\title{
Poincaré Map and Periodic Solutions of First-Order Impulsive Differential Equations on Moebius Stripe
}

\author{
Yefeng He and Yepeng Xing \\ Department of Mathematics, Shanghai Normal University, Shanghai 200234, China \\ Correspondence should be addressed to Yepeng Xing; ypxing-jason@hotmail.com
}

Received 12 December 2012; Accepted 1 January 2013

Academic Editor: Yonghui Xia

Copyright (C) 2013 Y. He and Y. Xing. This is an open access article distributed under the Creative Commons Attribution License, which permits unrestricted use, distribution, and reproduction in any medium, provided the original work is properly cited.

\begin{abstract}
This paper is mainly concerned with the existence, stability, and bifurcations of periodic solutions of a certain scalar impulsive differential equations on Moebius stripe. Some sufficient conditions are obtained to ensure the existence and stability of oneside periodic orbit and two-side periodic orbit of impulsive differential equations on Moebius stripe by employing displacement functions. Furthermore, double-periodic bifurcation is also studied by using Poincaré map.
\end{abstract}

\section{Introduction}

Many systems in physics, chemistry, biology, and information science have impulsive dynamical behavior due to abrupt jumps at certain instants during the evolving processes. This complex dynamical behavior can be modeled by impulsive differential equations. The theory of impulsive differential systems has been developed by numerous mathematicians (see [1-9]). As to the stability theory and boundary value problems to impulsive differential equations, There have been extensive studies in this area. However, there are very few works on the qualitative theory of impulsive differential equations and impulsive semidynamical systems. Recently, Bonotto and Federson have given a version of the PoincaréBendixson Theorem for impulsive semidynamical systems in $[10,11]$. As it is known, the method of Poincaré map plays an important role in the research of qualitative theory and is a natural means to study the existence of periodic solutions and its asymptotic stability. However, due to the complexity of the associated impulsive dynamic models, this approach has only been applied successfully to Raibert's one-legged-hopper (see [12-14]) predator-prey models (see [15-18]), and so forth. The bifurcation theory for ordinary differential equations or smooth systems appeared during the last decades (see, e.g., [19]); however, little is known about the bifurcation theory of impulsive differential equations due to its complexity (see [20]). In this paper, we mainly study a certain scalar impulsive differential equations on Moebius stripe undergoing impulsive effects at fixed time:

$$
\begin{gathered}
\frac{d x}{d t}=f(t, x), \quad t \neq \tau_{k}, \\
\left.\Delta x\right|_{t=\tau_{k}}=I_{k}(x), \quad k \in \mathbb{Z}^{+},
\end{gathered}
$$

where $0 \leq \tau_{k}<\tau_{k+1}, k \in \mathbb{Z}^{+}$are fixed with $\tau_{k} \rightarrow+\infty$ as, $k \rightarrow+\infty$, and $\left.\Delta x\right|_{t=\tau_{k}}=x\left(\tau_{k}^{+}\right)-x\left(\tau_{k}\right)$. Hu and Han (see [20]) investigated the existence of periodic solutions and bifurcations of (1) under the assumptions that $f(t, x)$ and $I_{k}(x)$ are periodic; that is, the following assumption holds.

$\left(\mathrm{H}^{*}\right)$ There exist a constant $T>0$, a positive integer $q$, and two mutual coprime positive integers $m$ and $n$ such that

$$
\begin{gathered}
f(t+T, x)=f(t, x), \quad \forall t \in \mathbb{R}^{+}, x \in \mathbb{R}, \\
I_{k+q}(x)=I_{k}(x), \quad \tau_{k+q}-\tau_{k}=T, \quad \forall k \in \mathbb{Z}^{+}, x \in \mathbb{R}, \\
m\left(t_{k+q}-t_{k}\right)=n T, \quad k \geq 1 .
\end{gathered}
$$

In this paper, we assume that the following conditions hold.

(H1) Assume that both $f(t, x)$ and $f_{x}(t, x)$ are continuous scalar functions on $\mathbb{R} \times \mathbb{R}, I_{k}(x): \mathbb{R} \rightarrow \mathbb{R}, k \in \mathbb{Z}$ are odd, continuous functions; that is, $I_{k}(-x)=-I_{k}(x), k \in \mathbb{Z}^{+}$. 
(H2) There exists a constant $T>0$, a positive integer $q$ such that

$$
\begin{gathered}
f(t+T,-x)=-f(t, x), \quad \forall t \in \mathbb{R}, x \in \mathbb{R}, \\
I_{k+q}(x)=I_{k}(x), \quad \tau_{k+q}-\tau_{k}=T, \quad \forall k \in \mathbb{Z}^{+}, x \in \mathbb{R} .
\end{gathered}
$$

From (H2), we have $f(t, x)$ is $2 T$ periodic and $\tau_{k+q}-\tau_{k}=2 T$. Hence assumption $\left(\mathrm{H}^{*}\right)$ holds naturally. However, we show some new and fruitful results of system (1) with the condition (H1)-(H2). For example, we obtain the existence and stability of $2 T$ periodic solutions to system (1) by double-periodic bifurcation.

This paper is organized as follows. In Section 2, for the sake of self-containedness of the paper, we present some basic definitions of impulsive differential equations. In Section 3, we describe the scalar impulsive differential equations on Moebius stripe and define the Poincaré map. Then we prove several essential lemmas and give sufficient conditions to ensure the existence and stability of one-side and two-side orbits of impulsive differential equation on Moebius stripe. In Section 4, we are mainly concerned with the doubleperiodic bifurcation impulsive differential equations on Moebius stripe.

\section{Preliminaries}

For the sake of self-containedness of the paper, we present the basic definitions and notations of the theory of impulsive differential equations we need (see $[1,2,8])$. We also include some fundamental results which are necessary for understanding the theory.

Let $\mathbb{R}, \mathbb{Z}$, and $\mathbb{Z}^{+}$be the sets of real numbers, integers, and positive integers, respectively. Denote by $\theta=\left\{\theta_{i}\right\}$ a strictly increasing sequence of real numbers such that the set $\mathfrak{A}$ of indexes $i$ is an interval in $\mathbb{Z}$.

Definition 1. A function $\phi: \mathbb{R} \rightarrow \mathbb{R}^{n}, n \in \mathbb{R}$, is from the set $P C(\mathbb{R}, \theta)$ if

(i) it is left continuous;

(ii) it is continuous, except, possibly, points of $\theta$, where it has discontinuities of the first kind.

The last definition means that if $\phi(t) \in P C(\mathbb{R}, \theta)$, then the right limit $\phi\left(\theta_{i}+\right)=\lim _{t \rightarrow \theta_{i}^{+}} \phi(t)$ exists and $\phi\left(\theta_{i}(-)\right)=\phi\left(\theta_{i}\right)$, where $\phi\left(\theta_{i}-\right)=\lim _{t \rightarrow \theta_{i}^{-}} \phi(t)$, for each $\theta_{i} \in \theta$.

Definition 2. A function $\phi: \mathbb{R} \rightarrow \mathbb{R}^{n}$ is from the set $P C^{1}(\mathbb{R}, \theta)$ if $\phi(t), \phi^{\prime}(t) \in P C(\mathbb{R}, \theta)$, where the derivative at points of $\theta$ is assumed to be the left derivative.

In what follows, in this section, $J \in \mathbb{R}$ is an interval in $\mathbb{R}$. For simplicity of notation, $\theta$ is not necessary a subset of $J$.

Definition 3. The solution $\phi(t)$ is stable if to any $\varepsilon>0$ and $t_{0} \in J$ there corresponds $\delta\left(t_{0}, \varepsilon\right)>0$ such that for any other solution $\psi(t)$ of (1) with $\left\|\phi\left(t_{0}\right)-\psi\left(t_{0}\right)\right\|<\delta\left(t_{0}, \varepsilon\right)$ we have $\|\phi(t)-\psi(t)\|<\varepsilon$ for $t \geq t_{0}$; the solution $\phi(t)$ is uniformly stable, if $\delta\left(t_{0}, \varepsilon\right)$ can be chosen independently of $t_{0}$.

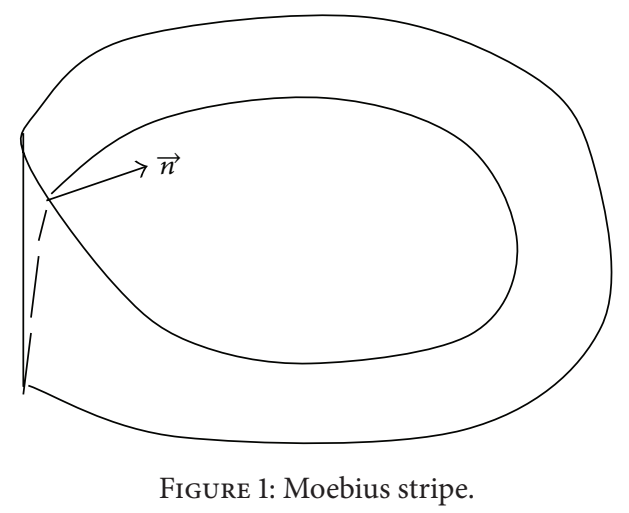

Definition 4. The solution $\phi(t)$ is asymptotically stable if it is stable in the sense of Definition 3 and there exists a positive number $\kappa\left(t_{0}\right)$ such that if $\psi(t)$ is any other solution of (1) with $\left\|\phi\left(t_{0}\right)-\psi\left(t_{0}\right)\right\|<\kappa\left(t_{0}\right)$, then $\|\phi(t)-\psi(t)\| \rightarrow 0$ as $t \rightarrow \infty$; if $\kappa\left(t_{0}\right)$ can be chosen to be independent of $t_{0}$ and $\phi(t)$ is uniformly stable, then $\phi(t)$ is said to be uniformly asymptotically stable.

Definition 5. The solution $\phi(t)$ is unstable if there exist numbers $\varepsilon_{0}>0$ and $t_{0} \in J$ such that for any $\delta>0$ there exists a solution $y_{\delta}(t),\left\|\phi\left(t_{0}\right)-y_{\delta}\left(t_{0}\right)\right\|<\delta$, of (1) such that either it is not continuable to $\infty$ or there exists a moment $t_{1}$, $t_{1}>t_{0}$ such that $\left\|\phi\left(t_{1}\right)-y_{\delta}\left(t_{1}\right)\right\| \geq \delta$.

For any $t_{0} \in \mathbb{R}$, we assume that there exists a $k \in \mathbb{Z}^{+}$, such that $\tau_{k-1}<t_{0} \leq \tau_{k}$; then the initial value problem (IVP) to first-order impulsive differential equations (1) is given as

$$
\begin{gathered}
\frac{d x}{d t}=f(t, x), \quad t \neq \tau_{k}, \\
\left.\Delta x\right|_{t=\tau_{k}}=I_{k}(x), \quad k \in \mathbb{Z}^{+}, \\
x\left(t_{0}^{+}\right)=x_{0} .
\end{gathered}
$$

In what followed, we use $x\left(t, t_{0}, x_{0}\right)$ to denote the solution of $\operatorname{IVP}(4)$.

In [20], Hu and Han investigated system (1) under the assumption $\left(\mathrm{H}^{*}\right)$ and obtained the following stability results for the periodic solutions.

Theorem 6 (see [20]). Let $x\left(t, t_{0}, x_{0}^{*}\right)$ be a periodic solution of system (1) with period T. If $0<\left|P^{\prime}\left(x_{0}^{*}\right)\right|<1(>1)$, then it is uniformly asymptotically stable (unstable), where $P\left(x_{0}\right)=$ $x\left(t_{0}+n T^{+}, t_{0}, x_{0}\right)$ is the Poincaré map of system (1).

\section{Poincaré Map and Periodic Solutions}

In this section, we describe the scalar impulsive differential equations on Moebius stripe and define the Poincaré map. Then we prove several essential lemmas and give sufficient conditions to ensure the existence and stability of oneside and two-side orbits (Figure 2) of impulsive differential equation on Moebius stripe. 
Lemma 7. Assume that conditions (H1), (H2) hold. Suppose that $x\left(t, t_{0}, x_{0}\right)$ is a solution of (1) satisfying initial value $x\left(t_{0}^{+}\right)=x_{0}$. Then $-x\left(t+T, t_{0}, x_{0}\right)$ is also a solution of (1), and

$$
-x\left(t+T, t_{0}, x_{0}\right)=x\left(t, t_{0},-x\left(t_{0}+T, t_{0}, x_{0}\right)\right), \quad t \in \mathbb{R} .
$$

Proof. Let $\varphi(t) \equiv-x\left(t+T, t_{0}, x_{0}\right), \psi(t) \equiv x\left(t, t_{0},-x\left(t_{0}+\right.\right.$ $\left.\left.T, t_{0}, x_{0}\right)\right)$. Then for $t \neq \tau_{k}, k \in \mathbb{Z}$, we have by (H2) that

$$
\begin{aligned}
\frac{d \varphi(t)}{d t} & =-\frac{d x\left(t+T, t_{0}, x_{0}\right)}{d t} \\
& =-f\left(t+T, x\left(t+T, t_{0}, x_{0}\right)\right) \\
& =f\left(t,-x\left(t+T, t_{0}, x_{0}\right)\right)=f(t, \varphi(t)) .
\end{aligned}
$$

For $t=\tau_{k}, k \in \mathbb{Z}^{+}$, it follows from (H1), (H2) that $\tau_{k}+T=$ $\tau_{k+q}, k \in \mathbb{Z}^{+}$and

$$
\begin{aligned}
\left.\Delta \varphi\right|_{t=\tau_{k}} & =-x\left(\tau_{k}+T^{+}, t_{0}, x_{0}\right)+x\left(\tau_{k}+T, t_{0}, x_{0}\right) \\
& =-x\left(\tau_{k+q}^{+}, t_{0}, x_{0}\right)+x\left(\tau_{k+q}, t_{0}, x_{0}\right) \\
& =-I_{k+q}\left(x\left(\tau_{k+q}, t_{0}, x_{0}\right)\right) \\
& =-I_{k}\left(x\left(\tau_{k+q}, t_{0}, x_{0}\right)\right)=-I_{k}\left(x\left(\tau_{k}+T, t_{0}, x_{0}\right)\right) \\
& =I_{k}\left(-x\left(\tau_{k}+T, t_{0}, x_{0}\right)\right)=I_{k}\left(\varphi\left(\tau_{k}\right)\right) .
\end{aligned}
$$

Thus, we proved that $\varphi(t) \equiv-x\left(t+T, t_{0}, x_{0}\right)$ is a solution of (1). On the other hand, it is obvious that

$$
\left.\varphi(t)\right|_{t=t_{0}}=-x\left(t_{0}+T, t_{0}, x_{0}\right)=\left.\psi(t)\right|_{t=t_{0}} .
$$

Hence, by uniqueness theorem we have that $\varphi(t) \equiv \psi(t), t \in$ $\mathbb{R}$. This completes the proof.

Let $D$ denotes the stripe area on the plain $\{(t, x) \mid(t, x) \in$ $\mathbb{R} \times \mathbb{R}\}$ between two lines $t=t_{0}$ and $t=t_{0}+T$; that is,

$$
D=\left\{(t, x) \mid t_{0} \leq t \leq t_{0}+T,-\infty<x<+\infty\right\} .
$$

Assume that $x\left(t, t_{0}, x_{0}\right)$ exists for all $t \in\left[t_{0},+\infty\right)$. Define $L_{0}=\left\{\left(t, x\left(t, t_{0}, x_{0}\right)\right) \mid t_{0} \leq t \leq t_{0}+T\right\}$. In general, we denote $L_{k}(k \geq 1)$ by

$$
L_{k}=\left\{\left(t, x\left(t, t_{0},-x_{k}\right)\right) \mid t_{0} \leq t \leq t_{0}+T\right\},
$$

where $x_{k}=x\left(t_{0}+T^{+}, t_{0},-x_{k-1}\right), t \geq t_{0}$.

It follows from Lemma 7 that $L_{k}$ has the form

$$
L_{k}=\left\{\left(t,(-1)^{k} x\left(t+k T, t_{0}, x_{0}\right)\right) \mid t_{0} \leq t \leq t_{0}+T\right\} .
$$

We now introduce an equivalence relation $\sim$ on $D$ such that for $(t, x),\left(t^{\prime}, x^{\prime}\right) \in D$

$$
(t, x) \sim\left(t^{\prime}, x^{\prime}\right) \text { iff }\left|t-t^{\prime}\right|=T, x=-x^{\prime} .
$$

Then we denote the corresponding quotient space by $M_{2}$. From geometric point of view, $M_{2}$ is obtained by considering

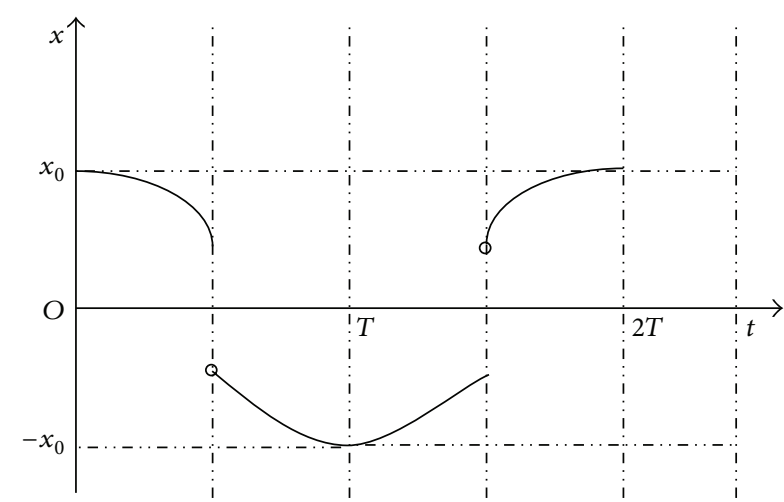

FIGURE 2: Figure of one-side and two-side orbits.

two elements $\left(t_{0}, x\right)$ and $\left(t_{0}+T,-x\right)$ on $D$ as the same point (or sticking $\left(t_{0}, x\right)$ and $\left(t_{0}+T,-x\right)$ together). Thus $M_{2}$ is a surface with only one side or the well-known Moebius stripe. Obviously, by Lemma 7 the union

$$
\bigcup_{k \in \mathbb{Z}^{+}} L_{k}=\bigcup_{k \in \mathbb{Z}^{+}}\left\{\left(t,(-1)^{k} x\left(t+k T, t_{0}, x_{0}\right)\right) \mid t_{0} \leq t \leq t_{0}+T\right\}
$$

define a flow on $M_{2}$. From this point of view, we call (1) satisfying ( $\mathrm{H} 1)$ and $(\mathrm{H} 2)$ an impulsive dynamical system on Moebius stripe (see Figure 1).

Definition 8 (Poincaré Map). Let $x\left(t, t_{0}, x_{0}\right)$ be the solution of (IVP) (4). Assume that there exists an interval $J$ such that for any $x_{0} \in J, x\left(t, t_{0}, x_{0}\right)$ exists on $\left[t_{0}, t_{0}+T\right]$. A map $P: J \rightarrow \mathbb{R}$ is called a Poincaré map of system (1) if for any $x_{0} \in J$

$$
P\left(x_{0}\right)=-x\left(t_{0}+T^{+}, t_{0}, x_{0}\right) .
$$

Definition 9. A closed curve $\gamma^{+}\left(x_{0}\right)$ is called a one-side periodic orbit on $M_{2}$ if $\gamma^{+}\left(x_{0}\right)=L_{0}$. And a closed curve $\gamma^{+}\left(x_{0}\right)$ is called a two-side periodic orbit on $M_{2}$ if $\gamma^{+}\left(x_{0}\right)=$ $L_{0} \cup L_{1} \neq L_{1}$.

From Definitions 8 and 9, we can easily prove the following assertion.

Lemma 10. One of following alternatives is valid:

(i) $\gamma^{+}\left(x_{0}\right)$ is a one-side periodic orbit;

(ii) $x_{0}$ is a fixed point of $P$; that is, $P\left(x_{0}\right)=x_{0}$;

(iii) $x\left(t+T, t_{0}, x_{0}\right)=-x\left(t, t_{0}, x_{0}\right), t \in \mathbb{R}$.

Proof. We prove it from (i) $\Rightarrow$ (ii) $\Rightarrow$ (iii) $\Rightarrow$ (i). Assume (i) is true; that is, $\gamma^{+}\left(x_{0}\right)$ is a one-side periodic orbit. Then by Definition 9 we have that

$$
-x\left(t_{0}+T^{+}, t_{0}, x_{0}\right)=x\left(t_{0}^{+}, t_{0}, x_{0}\right)=x_{0},
$$

that is, $P\left(x_{0}\right)=x_{0}$. Hence (ii) is valid. 
Next, we suppose that (ii) is fulfilled; that is, $-x\left(t_{0}+\right.$ $\left.T^{+}, t_{0}, x_{0}\right)=x_{0}$. Then by Lemma 7 we know

$$
\begin{aligned}
x\left(t+T, t_{0}, x_{0}\right) & =x\left(t, t_{0},-x\left(t_{0}+T^{+}, t_{0}, x_{0}\right)\right) \\
& =-x\left(t, t_{0}, x_{0}\right) .
\end{aligned}
$$

Thus, (iii) is proved.

Finally, if (iii) is true, then $x\left(t_{0}+T^{+}, t_{0}, x_{0}\right)=-x_{0}$. By the uniqueness of solution of IVP (4), we know

$$
\begin{array}{r}
x\left(t, t_{0},-x\left(t_{0}+T^{+}, t_{0}, x_{0}\right)\right)=x\left(t, t_{0}, x_{0}\right), \\
t \in\left[t_{0}, t_{0}+T\right] .
\end{array}
$$

Thus we obtain that $\gamma^{+}\left(x_{0}\right)$ is a one-side periodic orbit. The proof is completed. lemma.

Similarly, as proof of Lemma 10, we have the following

Lemma 11. One of following alternatives is valid:

(i) $\gamma^{+}\left(x_{0}\right)$ is a two-side periodic orbit;

(ii) $x_{0}$ is a 2-periodic point of $P$; that is, $P\left(x_{0}\right) \neq x_{0}$, $P^{2}\left(x_{0}\right)=x_{0}$

(iii) $x\left(t+2 T, t_{0}, x_{0}\right)=x\left(t, t_{0}, x_{0}\right), t \in \mathbb{R}$. And there exists a $t_{0}$, such that $x\left(t_{0}+T^{+}, t_{0}, x_{0}\right) \neq-x_{0}$.

Remark 12. From Lemmas 10 and 11, we see that a one-side periodic orbit must be a two-side periodic orbit since

$$
P\left(x_{0}\right)=x_{0} \quad \text { implies } P^{2}\left(x_{0}\right)=P\left(P\left(x_{0}\right)\right)=P\left(x_{0}\right)=x_{0} .
$$

Nevertheless, the converse is not true.

From Remark 12, we give the definition of stability of the mentioned orbits.

Definition 13. Let $\gamma^{+}\left(x_{0}\right)$ be a periodic orbit of system (1) (one-side or two-side). Then $\gamma^{+}\left(x_{0}\right)$ of system (1) is called stable (asymptotically stable or unstable) if $\gamma^{+}\left(x_{0}\right)$ as a $2 T$ periodic solution is stable (asymptotically stable or unstable).

Theorem 14. Assume $x\left(t, t_{0} x_{0}\right)$ is the solution of IVP (4) and let $z(t)=\partial x\left(t, t_{0}, x_{0}\right) / \partial x_{0}$. Then $z(t)$ is a solution to the following IVP of impulsive differential equations:

$$
\begin{gathered}
\frac{d z}{d t}=f_{x}(t, x) z, \quad t \neq t_{k}, \\
\left.\Delta z\right|_{t=\tau_{k}}=I_{k}^{\prime}(x), \quad k \in \mathbb{Z}^{+}, \\
z\left(t_{0}\right)=1 .
\end{gathered}
$$

Proof. Let $J=\left(t_{0},+\infty\right)$ and $J_{k}=\left(\tau_{k-1}, \tau_{k}\right], k \in \mathbb{Z}^{+}$. Without losing generality, we assume that $t_{0} \in J_{j}$ for some $j \geq 1$. The solution of IVP

$$
\begin{gathered}
\frac{d x}{d t}=f(t, x), \\
x\left(t_{0}^{+}\right)=x_{0}
\end{gathered}
$$

can be expressed as

$$
x\left(t, t_{0}, x_{0}\right)=x_{0}+\int_{t_{0}}^{t} f\left(s, x\left(s, t_{0}, x_{0}\right)\right) d s .
$$

Differentiate between both sides of the above equation with respect to $x_{0}$, we have

$$
\frac{\partial x\left(t, t_{0}, x_{0}\right)}{\partial x_{0}}=1+\int_{t_{0}}^{t} f_{x}\left(s, x\left(s, t_{0}, x_{0}\right)\right) \cdot \frac{\partial x\left(s, t_{0}, x_{0}\right)}{\partial x_{0}} d s
$$

Let $z(t)=\partial x\left(t, t_{0}, x_{0}\right) / \partial x_{0}$, then for $t \in\left[t_{0}, \tau_{j}\right), z(t)$ is the solution of IVP to ordinary differential equation

$$
\begin{gathered}
\frac{d z}{d t}=f_{x}(t, x) z, \quad t \neq t_{k}, \\
z\left(t_{0}\right)=1 .
\end{gathered}
$$

Thus

$$
z(t)=\exp \int_{t_{0}}^{t} f_{x}\left(s, x\left(s, t_{0}, x_{0}\right)\right) d s
$$

Since $z(t)$ is left continuous on $\left[t_{0}, \infty\right)$, we have

$$
z\left(\tau_{j}\right)=\exp \int_{t_{0}}^{\tau_{j}} f_{x}\left(s, x\left(s, t_{0}, x_{0}\right)\right) d s
$$

For $t \in J_{j+1}, x\left(t, t_{0}, x_{0}\right)$ is a solution of system

$$
\begin{gathered}
\frac{d x}{d t}=f(t, x), \\
x\left(\tau_{j}\right)=x_{1},
\end{gathered}
$$

where $x_{1}=x\left(\tau_{j^{+}}, t_{0}, x_{0}\right)=x\left(\tau_{j}, t_{0}, x_{0}\right)+I_{j}\left(x\left(\tau_{j}, t_{0}, x_{0}\right)\right)$. Thus, we have

$$
\begin{aligned}
x\left(t, t_{0}, x_{0}\right) & \equiv x\left(t, \tau_{j}, x_{1}\right) \\
& =x_{1}+\int_{\tau_{j}}^{t} f\left(s, x\left(s, \tau_{j}, x_{1}\right)\right) d s, \quad t \in J_{j+1} .
\end{aligned}
$$

Similarly, we have $t \in\left(\tau_{j}, \tau_{j+1}\right)$,

$$
\begin{aligned}
\frac{\partial x\left(t, t_{0}, x_{0}\right)}{\partial x_{1}} & =\frac{\partial x\left(t, \tau_{j}, x_{1}\right)}{\partial x_{1}} \\
& =\exp \int_{\tau_{j}}^{t} f_{x}\left(s, x\left(s, \tau_{j}, x_{1}\right)\right) d s \\
& =\exp \int_{\tau_{j}}^{t} f_{x}\left(s, x\left(s, t_{0}, x_{0}\right)\right) d s .
\end{aligned}
$$


Note

$$
\begin{aligned}
\frac{\partial x_{1}}{\partial x_{0}}= & \frac{\partial\left[x\left(\tau_{j}, t_{0}, x_{0}\right)+I_{j}\left(x\left(\tau_{j}, t_{0}, x_{0}\right)\right)\right]}{\partial x\left(\tau_{j}, t_{0}, x_{0}\right)} \\
& \cdot \frac{\partial x\left(\tau_{j}, t_{0}, x_{0}\right)}{\partial x_{0}} \\
= & \left(1+I_{j}^{\prime}\left(x\left(\tau_{j}, t_{0}, x_{0}\right)\right)\right) \frac{\partial x\left(\tau_{j}, t_{0}, x_{0}\right)}{\partial x_{0}} .
\end{aligned}
$$

We obtain for $t \in\left(\tau_{j}, \tau_{j+1}\right)$ that

$$
\begin{aligned}
\frac{\partial x\left(t, t_{0}, x_{0}\right)}{\partial x_{0}}= & \frac{\partial x\left(t, \tau_{j}, x_{1}\right)}{\partial x_{0}} \\
= & \left(1+I_{j}^{\prime}\left(x\left(\tau_{j}, t_{0}, x_{0}\right)\right)\right) \\
& \cdot \exp \int_{t_{0}}^{t} f_{x}\left(s, x\left(s, t_{0}, x_{0}\right)\right) d s .
\end{aligned}
$$

Deducing in a similar way, we get

$$
\begin{aligned}
\frac{\partial x\left(t, t_{0}, x_{0}\right)}{\partial x_{0}}= & \prod_{t_{0}<\tau_{k} \leq t}\left(1+I_{k}^{\prime}\left(x\left(\tau_{j}, t_{0}, x_{0}\right)\right)\right) \\
& \cdot \exp \int_{t_{0}}^{t} f_{x}\left(s, x\left(s, t_{0}, x_{0}\right)\right) d s,
\end{aligned}
$$

where $t \in J$. Then the proof is completed.

By Definitions 9 and (31), we conclude the following assertion.

Corollary 15. Assume that conditions (H1), (H2) hold. Then

$$
\begin{aligned}
P^{\prime}\left(x_{0}\right)= & -\prod_{t_{0}<\tau_{k} \leq t_{0}+T}\left(1+I_{k}^{\prime}\left(x\left(\tau_{k}, t_{0}, x_{0}\right)\right)\right) \\
& \cdot \exp \int_{t_{0}}^{t_{0}+T} f_{x}\left(t, x\left(t, t_{0}, x_{0}\right)\right) d t .
\end{aligned}
$$
has

As usual, one uses the notion $P^{2}\left(x_{0}\right)=P\left(P\left(x_{0}\right)\right)$. Then one

$$
\begin{aligned}
{\left[P^{2}\left(x_{0}\right)\right]^{\prime}=} & \prod_{t_{0}<\tau_{k} \leq t_{0}+2 T}\left(1+I_{k}^{\prime}\left(x\left(\tau_{k}, t_{0}, x_{0}\right)\right)\right) \\
& \cdot \exp \int_{t_{0}}^{t_{0}+2 T} f_{x}\left(t, x\left(t, t_{0}, x_{0}\right)\right) d t .
\end{aligned}
$$

Definition 16. $x_{0}$ is called a hyperbolic fixed point of $P$ if $x_{0}=P\left(x_{0}\right)$ and $P^{\prime}\left(x_{0}\right) \neq-1$; the corresponding one-side periodic orbit $\gamma^{+}\left(x_{0}\right)$ is called hyperbolic one-side periodic orbit. If $\gamma^{+}\left(x_{0}\right)$ is a two-side periodic orbit with $\left(P^{2}\right)^{\prime}\left(x_{0}\right) \neq 1$, then we call $\gamma^{+}\left(x_{0}\right)$ a hyperbolic two-side periodic orbit.
Theorem 17. Assume that the conditions (H1), (H2) hold. Let $\gamma^{+}\left(x_{0}\right)$ be a periodic orbit of system (1) and $I_{k}^{\prime}\left(x\left(\tau_{k}, t_{0}, x_{0}\right)\right) \neq-$ 1. Then (i) $\int_{t_{0}}^{t_{0}+2 T} f_{x}\left(t, x\left(t, t_{0}, x_{0}\right)\right) d t<-\sum_{t_{0}<\tau_{k} \leq t_{0}+2 T} \ln \mid 1+$ $I_{k}^{\prime}\left(x\left(\tau_{k}, t_{0}, x_{0}\right)\right) \mid$ implies $\gamma^{+}\left(x_{0}\right)$ is asymptotically stable,

(ii) $\int_{t_{0}}^{t_{0}+2 T} f_{x}\left(t, x\left(t, t_{0}, x_{0}\right)\right) d t>-\sum_{t_{0}<\tau_{k} \leq t_{0}+2 T} \ln \mid 1+$ $I_{k}^{\prime}\left(x\left(\tau_{k}, t_{0}, x_{0}\right)\right) \mid$ implies $\gamma^{+}\left(x_{0}\right)$ is unstable.

Proof. If $\gamma^{+}\left(x_{0}\right)$ is a two-side periodic orbit; that is, $x\left(t, t_{0}, x_{0}\right)$ is a $2 T$ periodic solution of (1). Since both (H1) and (H2) hold, we know that (1) is a periodic impulsive differential equation. Then by (33) and Theorem 6, the conclusion is straightforward.

Example 18. Consider the linear periodic impulsive differential equations on Moebius stripe as follows:

$$
\begin{aligned}
& \frac{d x}{d t}=a(t) x+b(t), \quad t \neq \tau_{k}, \\
& \left.\Delta x\right|_{t=\tau_{k}}=c_{k} x\left(\tau_{k}\right), \quad k \in \mathbb{Z}^{+},
\end{aligned}
$$

where $\tau_{k}<\tau_{k+1}(k \geq 1), \tau_{k} \rightarrow+\infty, k \rightarrow+\infty, c_{k} \neq-1$ and there exists a constant $T>0$, a positive integer $q$, such that the following conditions are satisfied:

$(\widetilde{\mathrm{H}} 1) a(t+T)=a(t)$ and $b(t+T)=-b(t)$ for $t \in \mathbb{R} ;$

$(\widetilde{\mathrm{H}} 2) a(t)$ and $b(t)$ are continuous;

$(\widetilde{\mathrm{H}} 3) c_{k+q}=c_{k}$, for all $k \in \mathbb{Z}^{+}$;

$(\widetilde{\mathrm{H}} 4) \tau_{k+q}-\tau_{k}=T$, for all $k \in \mathbb{Z}^{+}$.

Assume that $x\left(t, t_{0}, x_{0}\right)$ is a one-side periodic solution of system (34), by the method of variation of constants formula (see $[1])$, we get

$$
\begin{aligned}
& x\left(t, t_{0}, x_{0}\right) \\
& =\prod_{t_{0}<\tau_{k} \leq t}\left(1+c_{k}\right) \\
& \quad \cdot \exp \int_{t_{0}}^{t} a(t) d t \\
& \quad \times\left[x_{0}+\int_{t_{0}}^{t} \exp \left(-\int_{t_{0}}^{s} a(u) d u\right) b(s) d s\right],
\end{aligned}
$$

$$
\begin{aligned}
& P\left(x_{0}\right) \\
& =-\prod_{t_{0}<\tau_{k} \leq t_{0}+T}\left(1+c_{k}\right) \\
& \quad \exp \int_{t_{0}}^{t_{0}+T} a(t) d t \\
& \quad \times\left[x_{0}+\int_{t_{0}}^{t_{0}+T} \exp \left(-\int_{t_{0}}^{t} a(u) d u\right) b(t) d t\right],
\end{aligned}
$$




$$
\begin{aligned}
& P^{2}\left(x_{0}\right) \\
& =\prod_{t_{0}<\tau_{k} \leq t_{0}+2 T}\left(1+c_{k}\right) \\
& \quad \cdot \exp \int_{t_{0}}^{t_{0}+2 T} a(t) d t \\
& \quad \times\left[x_{0}+\int_{t_{0}}^{t_{0}+2 T} \exp \left(-\int_{t_{0}}^{t} a(u) d u\right) b(t) d t\right] .
\end{aligned}
$$

Let $A=\prod_{t_{0}<\tau_{k} \leq t_{0}+T}\left(1+c_{k}\right) \cdot \exp \int_{t_{0}}^{t_{0}+T} a(t) d t$; therefore, we have the following theorem.

\section{Theorem 19. Suppose that $(\widetilde{H} 1-\widetilde{H} 4)$ are satisfied, then}

(i) there exists a unique one-side periodic orbit for system (34) if $A \neq-1$, which is asymptotically stable (unstable) provided $0<|A|<1(|A|>1)$,

(ii) if $A^{2} \neq 1$, (34) has no two-side periodic orbit. If $A=1$ all the trajectories are two-side periodic orbits expect for a unique one-side periodic orbit.

Proof. For the sake of convenience, we denote

$$
\begin{aligned}
& B_{1}=\int_{t_{0}}^{t_{0}+T} \exp \left(-\int_{t_{0}}^{t} a(u) d u\right) b(t) d t \\
& B_{2}=\int_{t_{0}}^{t_{0}+2 T} \exp \left(-\int_{t_{0}}^{t} a(u) d u\right) b(t) d t .
\end{aligned}
$$

Then

$$
P\left(x_{0}\right)=-A\left(x_{0}+B_{1}\right), \quad P^{2}\left(x_{0}\right)=A^{2}\left(x_{0}+B_{2}\right) .
$$

Obviously, $P\left(x_{0}\right)=x_{0}$ has a unique solution for any $x_{0} \in \mathbb{R}$ if $A \neq-1$, and $P^{2}\left(x_{0}\right)=x_{0}$ has a unique solution for any $x_{0} \in \mathbb{R}$ if $A^{2} \neq 1$. Observing that any two-side periodic orbit obtained under the assumption $A^{2} \neq 1$ must be a one-side periodic orbit since $A^{2} \neq 1$ implies $A \neq-1$, together with Remark 12, we have (34) has no two-side periodic orbit.

It follows from (36) that $P^{\prime}\left(x_{0}\right)=-A$. Then by Theorem 6 we have the one-side orbit is asymptotically stable (unstable) provided $0<|A|<1(|A|>1)$. have

Next, let $A=1$. By taking (36) and (37) into account, we

$$
\begin{aligned}
P\left(x_{0}\right) & =-x_{0}-\int_{t_{0}}^{t_{0}+T} \exp \left(-\int_{t_{0}}^{t} a(u) d u\right) b(t) d t \\
& \equiv-x_{0}-B_{1} \\
P^{2}\left(x_{0}\right) & =x_{0}+\int_{t_{0}}^{t_{0}+2 T} \exp \left(-\int_{t_{0}}^{t} a(u) d u\right) b(t) d t \\
& \equiv x_{0}+B_{2} .
\end{aligned}
$$

Suppose that $P$ has a unique fixed point $x_{0}^{*}=-B_{1} / 2$, from the above we have $-B_{1} / 2+B_{2}=-B_{1} / 2$, then $B_{2} \equiv 0$ and
$P^{2}\left(x_{0}\right)=x_{0}$. So by taking Lemma $11, \gamma^{+}\left(x_{0}\right)$ is a two-side periodic orbit if $x_{0} \neq x_{0}^{*}$.

The proof is ended.

Remark 20. If $c_{k} \equiv 0, k \in \mathbb{Z}^{+}$, in (34); that is, (34) reduces to an ordinary differential equation. We see that $A=\exp \int_{t_{0}}^{t_{0}+T} a(t) d t$. Hence $A \neq-1$ holds automatically, and therefore (34) always has a unique one-side periodic orbit.

Corollary 21. Let $(\widetilde{H} 1-\widetilde{H} 4)$ be fulfilled and $A=1$. Then

$$
B_{2}=\int_{t_{0}}^{t_{0}+2 T} \exp \left(-\int_{t_{0}}^{t} a(u) d u\right) b(t) d t=0 .
$$

Now we are in position to consider nonlinear impulsive system on Meobius stripe. To explore the uniqueness of oneside periodic orbit, we induce the following condition.

(H3) Operator $B_{k}: \mathbb{R} \rightarrow \mathbb{R}, B_{k}(x)=x+I_{k}(x)$ is strictly increasing, for all $k \in \mathbb{Z}^{+}$.

Theorem 22. Suppose that conditions (H1)-(H3) hold, then

(i) system (1) has at most one one-side periodic orbit;

(ii) if any solution $x\left(t, t_{0}, x_{0}\right)$ of (1) with $\left|x_{0}\right| \leq|P(0)|$ is well defined on $t \in\left[t_{0}, t_{0}+T\right]$, then system (1) must has a unique one-side periodic orbit.

Proof. We first prove that system (1) cannot have two oneside periodic orbits. Suppose $\gamma_{1}^{+}\left(x_{0}^{*}\right): x=x\left(t, t_{0}, x_{0}^{*}\right), t \in$ $\left[t_{0}, t_{0}+T\right]$ and $\gamma_{2}^{+}\left(x_{0}\right): x=x\left(t, t_{0}, x_{0}\right), t \in\left[t_{0}, t_{0}+T\right]$ are two one-side periodic orbits system (1). Then

$$
x\left(t_{0}+T, t_{0}, x_{0}^{*}\right)=-x_{0}^{*}, \quad x\left(t_{0}+T, t_{0}, x_{0}\right)=-x_{0} .
$$

Without losing generality, we assume $x_{0}>x_{0}^{*}$, then it follows from uniqueness theorem of ordinary differential equations that $x=x\left(t, t_{0}, x_{0}\right)$ and $x\left(t, t_{0}, x_{0}^{*}\right)$ cannot intersect when $t$ is not an impulsive time. Therefore we have

$$
x\left(t, t_{0}, x_{0}\right)>x\left(t, t_{0}, x_{0}^{*}\right), \quad t_{0} \leq t \leq \tau_{1} .
$$

Note $B_{k}(x)=x+I_{k}(x)$ is strictly increasing, we get

$$
x\left(\tau_{1}^{+}, t_{0}, x_{0}\right)>x\left(\tau^{+}, t_{0}, x_{0}^{*}\right) .
$$

In a similar way, we can prove that $x\left(t, t_{0}, x_{0}\right)>x\left(t, t_{0}, x_{0}^{*}\right)$, $t_{0} \leq t \leq t_{0}+T$. That is, the curve $\left\{(t, x) \mid x=x\left(t, t_{0}, x_{0}\right)\right.$, $\left.t_{0} \leq t \leq t_{0}+T\right\}$ always stays above curve $\{(t, x) \mid x=$ $\left.x\left(t, t_{0}, x_{0}^{*}\right), t_{0} \leq t \leq t_{0}+T\right\}$. This contradicts (42). We put it in another way that

$$
x_{0}>x_{0}^{*} \Longrightarrow P\left(x_{0}\right)>P\left(x_{0}^{*}\right) .
$$

Thus, (1) has at most a one-side periodic orbit.

Further, let the solution $x\left(t, t_{0}, x_{0}\right)$ of system (1) be all defined on $t \in\left[t_{0}, t_{0}+T\right]$. If $P(0)=0$, the conclusion is proved. We assume that $P(0)>0$, then we know $P\left(x_{0}\right)<P(0)$ if $0<x_{0} \leq P(0)$. Note

$$
P^{2}(0)-P(0)=P(P(0))-P(0)<P(0)-P(0)=0 .
$$




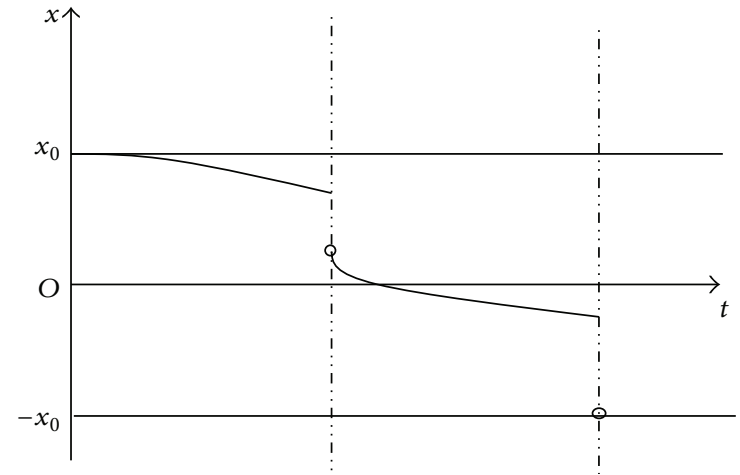

Figure 3: A one-side periodic orbit.

We obtain that $P\left(x_{0}\right)-x_{0}$ have opposite signs between $x_{0}=0$ and $x_{0}=P(0)$, and then it follows from the continuity of $P$ that there exists $x_{0}^{*} \in(0, P(0))$ such that $P\left(x_{0}^{*}\right)=x_{0}^{*}$. Similarly, we can prove $P$ has a fixed point in the case of $P(0)<0$. The proof is completed.

Theorem 23. Assume that conditions (H1)-(H3) hold. Furthermore, suppose there exists a positive number $N$ such that

$$
\begin{gathered}
f(t, N) \leq 0, \quad f(t,-N) \geq 0, \quad t \in\left[t_{0}, t_{0}+T\right], \\
-2 N \leq I_{k}(N) \leq 0, \quad \forall k \in \mathbb{Z}^{+} .
\end{gathered}
$$

Then (1) has a unique one-side periodic orbit.

Proof. From (47) we have that $x\left(t, t_{0}, x_{0}\right)$ will stay inside $[-N, N]$ for $t \neq \tau_{k}, k \in \mathbb{Z}^{+}$. On the other hand, by (H3), we have that $-N+I_{k}(-N) \leq x\left(\tau_{k}\right)+I_{k}\left(x\left(\tau_{k}\right)\right) \leq N+I_{k}(N)$ for $-N \leq x\left(\tau_{k}\right) \leq N$. Then it follows from (48) that

$$
\begin{gathered}
-N \leq N+I_{k}(N) \leq N \leq N \\
-N \leq-N+I_{k}(-N)=-N-I_{k}(N) \leq N
\end{gathered}
$$

(see Figure 3).

Thus,

$$
|P(0)|=\left|-x\left(t_{0}+T, 0\right)\right|=\left|x\left(t_{0}+T, 0\right)\right| \leq N
$$

This implies that $P\left(x_{0}\right)$ is well defined for $\left|x_{0}\right| \leq|P(0)|$. By Theorem 22, we obtain that (1) has a unique one-side periodic orbit.

\section{Double-Period Bifurcation}

In this section, we mainly discuss the bifurcation on periodic orbits. If system (1) has a one-side periodic orbit, without losing generality, we may assume that $f(t, 0)=0$; that is, $x=0$ is the one-side periodic orbit. Actually, if $x(t)$ is a one-side periodic orbit, then we let $y=x-x(t)$; therefore there exists a transformation of system (1) that

$$
\begin{gathered}
\frac{d y}{d t}=f(t, y+x(t))-f(t, x(t)) \equiv g(t, y), \quad t \neq \tau_{k}, \\
\left.\Delta y\right|_{t=\tau_{k}}=I_{k}\left(y+x\left(\tau_{k}\right)\right)-I_{k}\left(x\left(\tau_{k}\right)\right) \equiv h_{k}(y), \quad k \in \mathbb{Z}^{+},
\end{gathered}
$$

By (H2) and Lemma 10, we know $g(t+T,-y)=-g(t, y)$, $g(t, 0)=0, h_{k+q}(y)=h_{k}(y)$, and $\tau_{k+q}-\tau_{k}=T$, for all $k \in \mathbb{Z}^{+}$.

Next, we consider the following perturbed system of system (1):

$$
\begin{gathered}
\frac{d x}{d t}=F(t, x, \varepsilon), \quad t \neq \tau_{k}, \\
\left.\Delta x\right|_{t=\tau_{k}}=\widetilde{I}_{k}\left(x\left(\tau_{k}\right), \varepsilon\right), \quad k \in \mathbb{Z}^{+},
\end{gathered}
$$

where $F: \mathbb{R} \times \mathbb{R} \times \mathbb{R} \rightarrow \mathbb{R}$ is $C^{3}$ with respect to $x$, continuously differentiable with respect to $\varepsilon . \widetilde{I}_{k}: \mathbb{R} \times \mathbb{R} \rightarrow \mathbb{R}$ is $C^{3}\left(k \in \mathbb{Z}^{+}\right)$with respect to $x$. Moreover, we suppose $F(t+T,-x, \varepsilon)=-F(t, x, \varepsilon), \widetilde{I}_{k}(x, \varepsilon)=\widetilde{I}_{k+q}(x, \varepsilon), \widetilde{I}_{k}(-x)=$ $-\widetilde{I}_{k}(x)$, for all $k \in \mathbb{Z}^{+}$, where $\tau_{k+q}-\tau_{k}=T$. For $\varepsilon=0$, we have $F(t, x, 0)=f(t, x), \widetilde{I}_{k}(x, 0)=I_{k}(x)$. These assumptions mean (H1) and (H2) hold for $F$ and $\widetilde{I}_{k}$, for all $k \in \mathbb{Z}^{+}$. Furthermore, assume that $x+\widetilde{I}_{k}(x, \varepsilon)$ is strictly increasing, then by Theorem 22 we have that system (52) has at most a one-side periodic orbit.

Suppose that (1) has a one-side periodic orbit and $f(t, 0)=0$. Then by using implicit function theorem in the Poincaré map of system (52), we know that system (52) has a one-side periodic orbit when $|\varepsilon|$ is sufficiently small. Now let $x^{*}(t, \varepsilon)$ be the solution of system (52) and $y(t, \varepsilon)=x(t, \varepsilon)-$ $x^{*}(t, \varepsilon)$. Then we can get a transformation of system (52):

$$
\begin{aligned}
\frac{d y}{d t}= & F\left(t, y+x^{*}, \varepsilon\right)-F\left(t, x^{*}, \varepsilon\right)=G(t, y, \varepsilon), \quad t \neq \tau_{k}, \\
\left.\Delta y\right|_{t=\tau_{k}}= & \widetilde{I}_{k}\left(y+x^{*}\left(\tau_{k}, \varepsilon\right), \varepsilon\right) \\
& -\widetilde{I}_{k}\left(x^{*}\left(\tau_{k}, \varepsilon\right), \varepsilon\right)=H_{k}(y, \varepsilon), \quad k \in \mathbb{Z}^{+} .
\end{aligned}
$$

By Taylor's formula, we have

$$
\begin{aligned}
G(t, y, \varepsilon)= & A_{1}(t, \varepsilon) y+A_{2}(t, \varepsilon) y^{2} \\
& +A_{3}(t, \varepsilon) y^{3}+o\left(y^{3}\right), \\
H_{k}(y, \varepsilon)= & B_{k 1}(\varepsilon) y+B_{k 3}(\varepsilon) y^{3}+o\left(y^{3}\right),
\end{aligned}
$$

where

$$
\begin{aligned}
A_{i}(t, \varepsilon) & =\frac{1}{i !} \frac{\partial^{i} F}{\partial x^{i}}\left(t, x^{*}, \varepsilon\right), \\
B_{k j}(\varepsilon) & =\frac{1}{j !} \frac{\partial^{j} \widetilde{I}_{k}}{\partial x^{j}}\left(x^{*}, \varepsilon\right), \\
A_{i}(t+T, \varepsilon) & =(-1)^{i-1} A_{i}(t, \varepsilon),
\end{aligned}
$$

for $k \geq 1, i=1,2,3 ; j=1,3$. 
If $\varepsilon=0$, then $x^{*}=0$. So $A_{i}(t, 0)=(1 / i !)\left(\partial^{i} f / \partial x^{i}\right)(t, 0)$ and $B_{k j}(0)=(1 / j !) I_{k}^{(j)}(0)$, for $k \geq 1, i=1,2,3, j=1,3$.

Suppose that $y\left(t, y_{0}, \varepsilon\right)\left(t \geq t_{0}\right)$ is the solution of system (53) with the initial value $y\left(t_{0}^{+}, y_{0}, \varepsilon\right)=y_{0}, P\left(y_{0}, \varepsilon\right)$ is the Poincaré map of system (53). Note

$$
\widetilde{P}\left(y_{0}, \varepsilon\right)=y\left(t_{0}+2 T^{+}, y_{0}, \varepsilon\right)=P^{2}\left(y_{0}, \varepsilon\right) .
$$

Without losing generality, let $x^{*}(t, \varepsilon)=0$ is a nonhyperbolic solution. That is, $P(0,0)=0$ and $\left(\partial P / \partial y_{0}\right)(0,0)=-1$.

Noting that $P(0, \varepsilon)=0$, then by Taylor's formula, we have

$$
P\left(y_{0}, \varepsilon\right)=\bar{A}_{1}(\varepsilon) y_{0}+\bar{A}_{2}(\varepsilon) y_{0}^{2}+\bar{A}_{3}(\varepsilon) y_{0}^{3}+o\left(y_{0}^{3}\right),
$$

where $\bar{A}_{1}(\varepsilon)=\left(\partial P / \partial y_{0}\right)(0, \varepsilon), \bar{A}_{2}(\varepsilon)=(1 / 2)\left(\partial^{2} P / \partial y_{0}^{2}\right)(0, \varepsilon)$, and $\bar{A}_{3}(\varepsilon)=(1 / 6)\left(\partial^{3} P / \partial y_{0}^{3}\right)(0, \varepsilon)$.

Theorem 24. Suppose that $f(t, 0)=0$ and $x=0$ is a oneside periodic orbit of system $(52)_{\varepsilon=0}$ with $P(0,0)=0$ and $\left(\partial P / \partial y_{0}\right)(0,0)=-1$. Let $a_{3}^{*}=(1 / 6) \widetilde{P}^{\prime \prime \prime}(0,0)$. If $a_{3}^{*} \neq 0$, then for $|\varepsilon|$ sufficiently small and $\left[\bar{A}_{1}(\varepsilon)+1\right] a_{3}^{*}>0(\leq 0)$ implies that system (52) has a unique (no) two-sides periodic orbit near $x=0$, except for a one-side periodic orbit $x^{*}(t, \varepsilon)$.

Proof. As before, we obtain that $\widetilde{P}^{\prime}(0, \varepsilon)=\left[P^{\prime}(0, \varepsilon)\right]^{2}=\bar{A}_{1}^{2}(\varepsilon)$ :

$$
\begin{aligned}
\widetilde{P}\left(y_{0}, \varepsilon\right)= & P\left(P\left(y_{0}, \varepsilon\right), \varepsilon\right) \\
= & \bar{A}_{1}(\varepsilon) P\left(y_{0}, \varepsilon\right)+\bar{A}_{2}(\varepsilon) P^{2}\left(y_{0}, \varepsilon\right) \\
& +\bar{A}_{3}(\varepsilon) P^{3}\left(y_{0}, \varepsilon\right)+o\left(P^{3}\left(y_{0}, \varepsilon\right)\right) \\
= & \bar{A}_{1}^{2}(\varepsilon) y_{0}+\left[\bar{A}_{1}^{2}(\varepsilon)+\bar{A}_{1}(\varepsilon)\right] \bar{A}_{2}(\varepsilon) y_{0}^{2} \\
& +\bar{A}_{1}\left[\bar{A}_{3}(\varepsilon)+2 \bar{A}_{2}^{2}(\varepsilon)+\bar{A}_{1}^{2}(\varepsilon) \bar{A}_{3}(\varepsilon)\right] y_{0}^{3} \\
& +o\left(y_{0}^{3}\right) .
\end{aligned}
$$

By our assumption, we have $\bar{A}_{1}(\varepsilon)=-1+\bar{A}_{1}^{\prime}(0) \varepsilon+o(\varepsilon)$. Therefore,

$$
\begin{aligned}
\tilde{d}\left(y_{0}, \varepsilon\right) & =\frac{P^{2}\left(y_{0}, \varepsilon\right)-y_{0}}{y_{0}} \\
& =d_{0}(\varepsilon)+d_{1}(\varepsilon) y_{0}+d_{2}(\varepsilon) y_{0}^{2}+o\left(y_{0}^{2}\right),
\end{aligned}
$$

where

$$
\begin{aligned}
d_{0}(\varepsilon) & =\bar{A}_{1}^{2}(\varepsilon)-1=-2\left[\bar{A}_{1}(\varepsilon)+1\right]+o\left(\left[\bar{A}_{1}(\varepsilon)+1\right]\right), \\
d_{1}(\varepsilon) & =\left[\bar{A}_{1}^{2}(\varepsilon)+\bar{A}_{1}(\varepsilon)\right] \bar{A}_{2}(\varepsilon) \\
& =O\left(\bar{A}_{1}(\varepsilon)+1\right)=-\bar{A}_{1}^{\prime}(0) \bar{A}_{2}(0) \varepsilon+o(\varepsilon), \\
d_{2}(\varepsilon) & =\bar{A}_{1}(\varepsilon)\left[\bar{A}_{3}(\varepsilon)+2 \bar{A}_{2}^{2}(\varepsilon)+\bar{A}_{1}^{2}(\varepsilon) \bar{A}_{3}(\varepsilon)\right] \\
& =-2\left[\bar{A}_{2}^{2}(0)+\bar{A}_{3}(0)\right]+o(1)=a_{3}^{*}+o(1) .
\end{aligned}
$$

By the implicit function theorem, there exists a unique function $y_{0}=y_{1}(\varepsilon), y_{1}(0)=0$ such that $\left(\partial \tilde{d} / \partial y_{0}\right)\left(y_{1}(\varepsilon), \varepsilon\right)=$ 0 . Therefore, for $|\varepsilon|$ sufficiently small, there is a unique extremal point $y_{0}=y_{1}(\varepsilon)$ near $x=0$. Moreover, the function $\widetilde{d}\left(y_{0}, \varepsilon\right)$ takes its minimum (maximum) $\bar{\Delta}(\varepsilon) \equiv \widetilde{d}\left(y_{1}(\varepsilon), \varepsilon\right)$ only if $a_{3}^{*}>0(<0)$ :

$$
\bar{\Delta}(\varepsilon)=d_{0}(\varepsilon)+o(\varepsilon)=\bar{A}_{1}^{2}(\varepsilon)-1=-2\left[\bar{A}_{1}(\varepsilon)+1\right]+o(\varepsilon) .
$$

Without loss of generality, we can let $a_{3}^{*}=d_{2}(0)>0$ and then $y_{0}=0$ is the minimum point of $d\left(y_{0}, 0\right)$. So there exists $\varepsilon_{0}>0$, such that

$$
\widetilde{d}\left( \pm \varepsilon_{0}, 0\right)>0, \quad \frac{\partial \widetilde{d}}{\partial y_{0}}\left(\varepsilon_{0}, 0\right)>0, \quad \frac{\partial \tilde{d}}{\partial y_{0}}\left(-\varepsilon_{0}, 0\right)<0,
$$

and for $\left|y_{0}\right| \leq \varepsilon_{0},\left(\partial^{2} \widetilde{d} / \partial y_{0}^{2}\right)\left(y_{0}, 0\right)>0$ exists. Therefore, there exists a $\delta_{0}$, such that, for $|\varepsilon| \leq \delta_{0}$, we have

$$
\tilde{d}\left( \pm \varepsilon_{0}, \varepsilon\right)>0, \quad \frac{\partial \widetilde{d}}{\partial y_{0}}\left(\varepsilon_{0}, \varepsilon\right)>0, \quad \frac{\partial \tilde{d}}{\partial y_{0}}\left(-\varepsilon_{0}, \varepsilon\right)<0 .
$$

For $|\varepsilon| \leq \delta_{0}$ and $\left|y_{0}\right| \leq \varepsilon_{0}$, we have

$$
\frac{\partial^{2} \widetilde{d}}{\partial y_{0}^{2}}\left(y_{0}, \varepsilon\right)>0
$$

From (64), for any $|\varepsilon| \leq \delta_{0}$, we have

$$
\bar{\Delta}(\varepsilon)=\min _{\left|y_{0}\right| \leq \varepsilon_{0}} \widetilde{d}\left(y_{0}, \varepsilon\right), \quad-\varepsilon_{0}<y_{1}(\varepsilon)<\varepsilon_{0} .
$$

And for $y_{0} \in\left(-\varepsilon_{0}, y_{1}(\varepsilon)\right)\left(\in\left(y_{1}(\varepsilon), \varepsilon_{0}\right)\right)$,

$$
\frac{\partial \widetilde{d}}{\partial y_{0}}\left(y_{0}, \varepsilon\right)<0(>0) \text {. }
$$

If $\bar{\Delta}(\varepsilon)>0$, then for all $|\varepsilon| \leq \delta_{0}$ and $\left|x_{0}\right|<\varepsilon_{0}$, we have $0<\bar{\Delta}(\varepsilon) \leq \widetilde{d}$.

If $\bar{\Delta}(\varepsilon)=0$, then $y_{0}=y_{1}(\varepsilon)$ is the unique solution of function $\tilde{d}$.

If $\bar{\Delta}(\varepsilon)<0$, then there exist a unique $y_{1}(\varepsilon)$ and a unique $y_{2}(\varepsilon)$, such that

$$
\widetilde{d}\left(y_{i}(\varepsilon), \varepsilon\right)=0, \quad \frac{\partial \widetilde{d}}{\partial y_{0}}\left(y_{i}(\varepsilon), \varepsilon\right) \neq 0, \quad i=1,2 .
$$

Thus system (52) has two (no) two-side periodic orbits if $a_{3}^{*} \bar{\Delta}(\varepsilon)<0(\geq 0)$. The conclusion is completed (see Figures $4,5$, and 6$)$. 


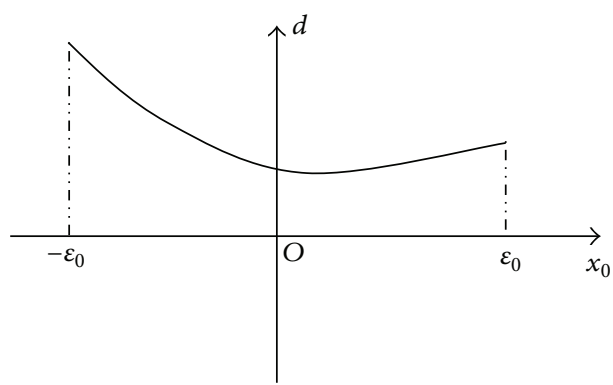

$\bar{\Delta}>0$

FIGURE 4

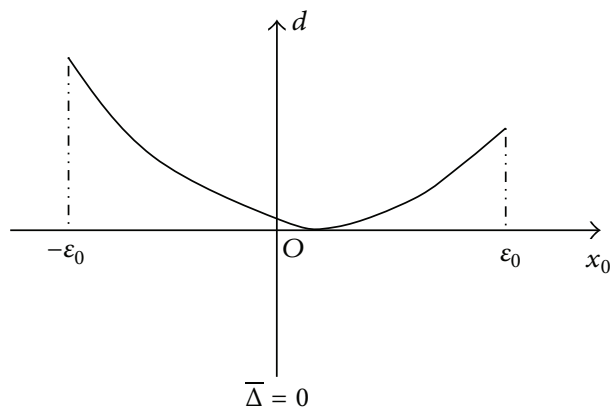

Figure 5

Now we shall calculate $\bar{A}_{1}(0)$ and $a_{3}^{*}$ in the simplest case, let $q=1$. For $q>1$ we can calculate them in the same way. In this case, $I_{k} \equiv I$ and $B_{k i}\left(y_{0}, \varepsilon\right)=B_{i}\left(y_{0}, \varepsilon\right), i=1$, 3. Suppose $y\left(t, y_{0}, \varepsilon\right)\left(t \leq t_{0}\right)$ is the solution to system (53) with initial value $y\left(t_{0}+, y_{0}, \varepsilon\right)=y_{0}$. For $y(t, 0, \varepsilon)=0$, let

$$
\begin{aligned}
y\left(t, y_{0}, \varepsilon\right)= & \varphi_{1}(t, \varepsilon) y_{0}+\varphi_{2}(t, \varepsilon) y_{0}^{2} \\
& +\varphi_{3}(t, \varepsilon) y_{0}^{3}+o\left(y_{0}^{3}\right), \quad t \leq t_{0} .
\end{aligned}
$$

Then for $t \in\left[t_{0}, t_{0}+T\right]$, taking $y\left(t, y_{0}, \varepsilon\right)$ into system (53), we can obtain $\varphi_{1}, \varphi_{2}$, and $\varphi_{3}$ satisfying the following equations:

$$
\begin{aligned}
\varphi_{1}^{\prime}(t, \varepsilon)= & A_{1}(t, \varepsilon) \varphi_{1}(t, \varepsilon) \\
\varphi_{2}^{\prime}(t, \varepsilon)= & A_{1}(t, \varepsilon) \varphi_{2}(t, \varepsilon)+A_{2}(t, \varepsilon) \varphi_{1}^{2}(t, \varepsilon) \\
\varphi_{3}^{\prime}(t, \varepsilon)= & A_{1}(t, \varepsilon) \varphi_{3}(t, \varepsilon)+2 A_{2}(t, \varepsilon) \varphi_{1}(t, \varepsilon) \varphi_{2}(t, \varepsilon) \\
& +\varphi_{3}(t, \varepsilon) \varphi_{1}^{3}(t, \varepsilon) .
\end{aligned}
$$

For $y\left(0, y_{0}, \varepsilon\right)=y_{0}$, we know

$$
\varphi_{1}(0, \varepsilon)=1, \quad \varphi_{2}(0, \varepsilon)=\varphi_{3}(0, \varepsilon)=0 .
$$

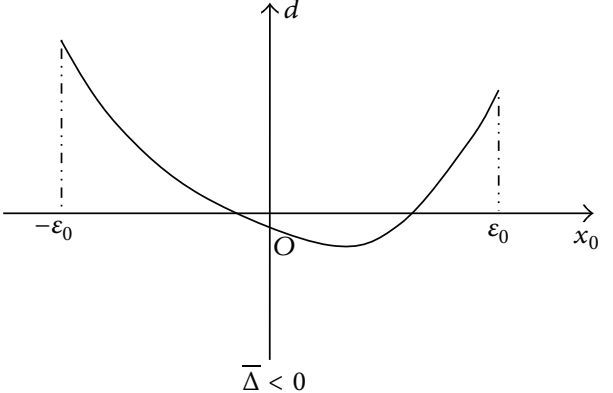

Figure 6

From (69) and (70), we have

$$
\begin{aligned}
& \varphi_{1}(t, \varepsilon)=\exp \int_{t_{0}}^{t} A_{1}(u, \varepsilon) d u \\
& \varphi_{2}(t, \varepsilon)=\varphi_{1}(t, \varepsilon) \int_{t_{0}}^{t} A_{2}(s, \varepsilon) \varphi_{1}(s, \varepsilon) d s \\
& \varphi_{3}(t, \varepsilon)=\varphi_{1}(t, \varepsilon) \\
& \quad \times \int_{t_{0}}^{t}\left[2 A_{2}(s, \varepsilon) \varphi_{2}(s, \varepsilon)+A_{3}(s, \varepsilon) \varphi_{1}^{2}(s, \varepsilon)\right] d s .
\end{aligned}
$$

For $t_{0}<t<t_{0}+T$, as we know, we get

$$
\begin{aligned}
y\left(t_{0}+T^{+}, y_{0}, \varepsilon\right)= & {\left[1+B_{1}(\varepsilon)\right] y\left(t_{0}+T, \varepsilon\right) } \\
& +B_{2}(\varepsilon) y^{2}\left(t_{0}+T, \varepsilon\right) \\
& +B_{3}(\varepsilon) y^{3}\left(t_{0}+T, \varepsilon\right) \\
& +o\left(y^{3}\left(t_{0}+T, \varepsilon\right)\right) \\
= & \varphi_{1}\left(t_{0}+T^{+}, \varepsilon\right) y_{0}+\varphi_{2}\left(t_{0}+T^{+}, \varepsilon\right) y_{0}^{2} \\
& +\varphi_{3}\left(t_{0}+T^{+}, \varepsilon\right) y_{0}^{3}+o\left(y_{0}^{3}\right),
\end{aligned}
$$

where

$$
\begin{aligned}
\varphi_{1}\left(t_{0}+T^{+}, \varepsilon\right)= & {\left[1+B_{1}(\varepsilon)\right] \varphi_{1}\left(t_{0}+T, \varepsilon\right), } \\
\varphi_{2}\left(t_{0}+T^{+}, \varepsilon\right)= & {\left[1+B_{1}(\varepsilon)\right] \varphi_{2}\left(t_{0}+T, \varepsilon\right) } \\
& +B_{2}(\varepsilon) \varphi_{1}^{2}\left(t_{0}+T, \varepsilon\right), \\
\varphi_{3}\left(t_{0}+T^{+}, \varepsilon\right)= & {\left[1+B_{1}(\varepsilon)\right] \varphi_{3}\left(t_{0}+T, \varepsilon\right) } \\
& +2 B_{2}(\varepsilon) \varphi_{1}\left(t_{0}+T, \varepsilon\right) \varphi_{2}\left(t_{0}+T, \varepsilon\right) \\
& +B_{3}(\varepsilon) \varphi^{3}\left(t_{0}+T, \varepsilon\right) .
\end{aligned}
$$

Clearly, $\bar{A}_{1}(\varepsilon)=-\varphi_{1}\left(t_{0}+T^{+}, \varepsilon\right), \bar{A}_{2}(\varepsilon)=-\varphi_{2}\left(t_{0}+T^{+}, \varepsilon\right)$, and $\bar{A}_{3}(\varepsilon)=-\varphi_{3}\left(t_{0}+T^{+}, \varepsilon\right)$. 
Moreover, we know

$$
\begin{aligned}
\int_{t_{0}}^{t_{0}+T} & A_{2}(s, \varepsilon) \varphi_{2}(s, \varepsilon) d s \\
= & \int_{t_{0}}^{t_{0}+T} A_{2}(s, \varepsilon) \varphi_{1}(s, \varepsilon) \\
& \times\left[\int_{t_{0}}^{s} A_{2}(u, \varepsilon) \varphi_{1}(u, \varepsilon) d u\right] d s \\
= & \frac{1}{2}\left[\int_{t_{0}}^{t_{0}+T} A_{2}(u, \varepsilon) \varphi_{1}(u, \varepsilon) d u\right]^{2}
\end{aligned}
$$

Denote $\Phi(\varepsilon)=\int_{t_{0}}^{t_{0}+T} A_{2}(t, \varepsilon) \varphi_{1}(t, \varepsilon) d t$ and $\phi_{1}(\varepsilon)=\varphi_{1}\left(t_{0}+\right.$ $T, \varepsilon)$. Then,

$$
\begin{gathered}
\varphi_{2}\left(t_{0}+T, \varepsilon\right)=\phi_{1}(\varepsilon) \Phi(\varepsilon) \\
\varphi_{3}\left(t_{0}+T, \varepsilon\right)=\phi_{1}(\varepsilon) \Phi^{2}(\varepsilon)+\int_{t_{0}}^{t_{0}+T} A_{3}(s, \varepsilon) \varphi_{1}^{2}(s, \varepsilon) d s .
\end{gathered}
$$

Then we can obtain

$$
\begin{aligned}
\bar{A}_{1}(\varepsilon)= & -\left[1+B_{1}(\varepsilon)\right] \exp \left(\int_{t_{0}}^{t_{0}+T} A_{1}(u, \varepsilon) d u\right) \\
\bar{A}_{2}(\varepsilon)= & -\left[1+B_{1}(\varepsilon)\right] \phi_{1}(\varepsilon) \Phi(\varepsilon) \\
\bar{A}_{3}(\varepsilon)= & -\left[1+B_{1}(\varepsilon)\right] \phi_{1}(\varepsilon) \\
& \times\left[\Phi^{2}(\varepsilon)+\int_{t_{0}}^{t_{0}+T} A_{3}(s, \varepsilon) \varphi_{1}^{2}(s, \varepsilon) d s\right] \\
& -B_{3}(\varepsilon) \phi_{1}^{3}(\varepsilon)
\end{aligned}
$$

For $\bar{A}_{1}(0)=-1$, we can have $\phi_{1}(0)=1 /\left(1+B_{1}(0)\right)$. Then

$$
\begin{gathered}
\bar{A}_{2}(0)=-\Phi(0) \\
\bar{A}_{3}(0)=-\Phi^{2}(0)-\frac{B_{3}(0)}{\left[1+B_{1}(0)\right]^{3}}-\Delta(0)
\end{gathered}
$$

where $\Delta(\varepsilon)=\int_{t_{0}}^{t_{0}+T} A_{3}(s, \varepsilon) \varphi_{1}^{2}(s, \varepsilon) d s$ $\int_{t_{0}}^{t_{0}+T} A_{3}(s, \varepsilon) \exp \left[2 \int_{t_{0}}^{s} A_{1}(u, \varepsilon) d u\right] d s$. Therefore,

$$
a_{3}^{*}=-2\left[\bar{A}_{2}^{2}(0)+\bar{A}_{3}(0)\right]=2 \Delta(0)+\frac{2 B_{3}(0)}{\left[1+B_{1}(0)\right]^{3}} \text {. }
$$

By considering (76)-(79), we can easily have the following theorem when $q=1$.
Theorem 25. Suppose that $f(t, 0)=0$ and $x=0$ is a oneside periodic solution of system $(52)_{\varepsilon=0}$ with $P(0,0)=0$ and $\left(\partial P / \partial y_{0}\right)(0,0)=-1$. Let

$$
\begin{aligned}
& \bar{A}_{1}(\varepsilon)=-\left(1+\widetilde{I}^{\prime}\left(x^{*}, \varepsilon\right)\right) \exp \int_{t_{0}}^{t_{0}+T} F_{x}\left(t, x^{*}, \varepsilon\right) d t, \\
& a_{3}^{*}=\frac{1}{6} \int_{t_{0}}^{t_{0}+2 T} f_{x}^{\prime \prime \prime}(s, 0) e^{2 \int_{t_{0}}^{s} f_{x}(u, 0) d u} d s+\frac{2 \widetilde{I}^{\prime \prime \prime}(0)}{\left[1+\widetilde{I}^{\prime}(0)\right]^{3}} .
\end{aligned}
$$

If $a_{3}^{*} \neq 0$, then for $|\varepsilon|$ sufficiently small, $\left[\bar{A}_{1}(\varepsilon)+1\right] a_{3}^{*}>0(\leq 0)$ implies that system (52) has a unique (no) two-side periodic orbit of near $x=0$, except for a one-side periodic orbit $x^{*}(t, \varepsilon)$.

By virtue of Theorem 25, we can have the following conclusion.

Corollary 26. (i) Let $\bar{A}_{1}(0)=-1, a_{3}^{*}>0(<0)$. Then $x=0$ is a nonhyperbolic one-side periodic orbit of system $(48)(\varepsilon=0)$, which is asymptotically stable (unstable). (ii) Let $\bar{A}_{1}(0)=-1$, $\left[\bar{A}_{1}(\varepsilon)+1\right] a_{3}^{*}>0,0<|\varepsilon| \ll 1$. Then $\left(\bar{A}_{1}(\varepsilon)+1\right)<0(>0)$, $x^{*}(t, \varepsilon)$ is a hyperbolic one-side periodic orbit of system (48) $(\varepsilon=0)$, which is asymptotically stable (unstable. Moreover, the two-side periodic orbit is unstable (asymptotically stable) near $x=0$.

Finally, we give an example to illustrate it.

Example 27. Consider

$$
\begin{gathered}
\frac{d x}{d t}=\varepsilon^{2} x+|\varepsilon|(\sin t) x^{2}-(1-2 \cos 2 t) x^{3}, \quad t \neq k \pi, \\
\left.\Delta x\right|_{t=k \pi}=-\varepsilon x(k \pi)+\left(b_{3}+\varepsilon\right) x^{3}(k \pi), \quad k \in \mathbb{Z}^{+},
\end{gathered}
$$

where $|\varepsilon|>0$. It is obvious that for $|\varepsilon|>0$ sufficiently small, $x-\varepsilon x+\left(b_{3}+\varepsilon\right) x^{3}$ is strictly increasing, and then $x^{*}(t, \varepsilon)=0$ is the unique $\pi$-periodic solution. By direct computation, we have $A_{1}(t, \varepsilon)=\varepsilon^{2}, A_{2}(t, \varepsilon)=|\varepsilon|(\sin t), A_{3}(t, \varepsilon)=2 \cos 2 t-1$, $B_{1}(\varepsilon)=-\varepsilon$, and $B_{3}(\varepsilon)=b_{3}+\varepsilon$. Therefore, $\bar{A}_{1}(\varepsilon)+1=(\varepsilon-$ 1) $e^{\varepsilon^{2} \pi}+1=\varepsilon+o(\varepsilon), a_{3}^{*}=2 b_{3}-\pi$. It follows from Theorem 25 that system (81) has two (no) $2 \pi$-periodic solution of near $x=0$ if $|\varepsilon|$ is sufficiently small and $a_{3}^{*} \varepsilon>0(\leq 0)$.

\section{Acknowledgments}

This work was supported by National Natural Science Foundation of China (no. 11271261), Shanghai Natural Science Foundation (no. 12ZR1421600), and Shanghai Educational Committee Project (10YZ74).

\section{References}

[1] M. Akhmet, Principles of Discontinuous Dynamical Systems, Springer, New York, NY, USA, 2010.

[2] D. D. Bainov and P. S. Simenov, Impulsive Differential Equations: Periodic Solutions and Applications, Longman Scientific and Technical, Harlow, UK, 1993. 
[3] S. Djebali, L. Górniewicz, and A. Ouahab, "First-order periodic impulsive semilinear differential inclusions: existence and structure of solution sets," Mathematical and Computer Modelling, vol. 52, no. 5-6, pp. 683-714, 2010.

[4] X. L. Fu, B. Q. Yan, and Y. S. Liu, Theory of Impulsive Differential System, Science Press, Beijing, China, 2005.

[5] V. Lakshmikantham, D. D. Baĭnov, and P. S. Simeonov, Theory of Impulsive Differential Equations, vol. 6 of Series in Modern Applied Mathematics, World Scientific Publishing, Singapore, 1989.

[6] Y. Liu, "Further results on periodic boundary value problems for nonlinear first order impulsive functional differential equations," Journal of Mathematical Analysis and Applications, vol. 327, no. 1, pp. 435-452, 2007.

[7] J. Li and J. Shen, "Periodic boundary value problems for impulsive differential-difference equations," Indian Journal of Pure and Applied Mathematics, vol. 35, no. 11, pp. 1265-1277, 2004.

[8] A. M. Samoilenko and N. A. Perestyuk, Impulsive Differential Equations, World Scientic Publishing, Singapore, 1995.

[9] J. Shen, "New maximum principles for first-order impulsive boundary value problems," Applied Mathematics Letters, vol. 16, no. 1, pp. 105-112, 2003.

[10] E. M. Bonotto, "LaSalle's theorems in impulsive semidynamical systems," Nonlinear Analysis: Theory, Methods \& Applications, vol. 71, no. 5-6, pp. 2291-2297, 2009.

[11] E. M. Bonotto and M. Federson, "Limit sets and the PoincaréBendixson theorem in impulsive semidynamical systems," Journal of Differential Equations, vol. 244, no. 9, pp. 2334-2349, 2008.

[12] J. W. Grizzle, G. Abba, and F. Plestan, "Asymptotically stable walking for biped robots: analysis via systems with impulse effects," IEEE Transactions on Automatic Control, vol. 46, no. 1, pp. 51-64, 2001.

[13] I. A. Hiskens, "Stability of hybrid system limit cycles: application to the Compass Gait Biped Robot," in Proceedings of the 40th lEEE Conference on Decision and Control, Oriando, Fla, USA, December 2001.

[14] B. Morris and J. W. Grizzle, "Hybrid invariant manifolds in systems with impulse effects with application to periodic locomotion in bipedal robots," IEEE Transactions on Automatic Control, vol. 54, no. 8, pp. 1751-1764, 2009.

[15] K. G. Dishlieva, "Differentiability of solutions of impulsive differential equations with respect to the impulsive perturbations," Nonlinear Analysis: Real World Applications, vol. 12, no. 6, pp. 3541-3551, 2011.

[16] G. Jiang and Q. Lu, "Impulsive state feedback control of a predator-prey model," Journal of Computational and Applied Mathematics, vol. 200, no. 1, pp. 193-207, 2007.

[17] L. Nie, Z. Teng, L. Hu, and J. Peng, "Qualitative analysis of a modified Leslie-Gower and Holling-type II predatorprey model with state dependent impulsive effects," Nonlinear Analysis: Real World Applications, vol. 11, no. 3, pp. 1364-1373, 2010.

[18] Z. Teng, L. Nie, and X. Fang, "The periodic solutions for general periodic impulsive population systems of functional differential equations and its applications," Computers \& Mathematics with Applications, vol. 61, no. 9, pp. 2690-2703, 2011.

[19] M. Han and S. Gu, Theory and Method of Nonlinear System, Science Press, Beijing, China, 2001.
[20] Z. Hu and M. Han, "Periodic solutions and bifurcations of firstorder periodic impulsive differential equations," International Journal of Bifurcation and Chaos in Applied Sciences and Engineering, vol. 19, no. 8, pp. 2515-2530, 2009. 


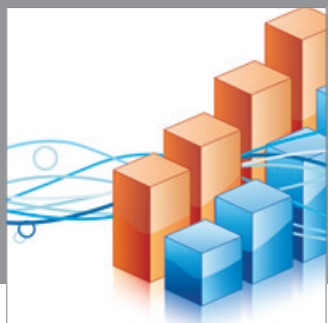

Advances in

Operations Research

mansans

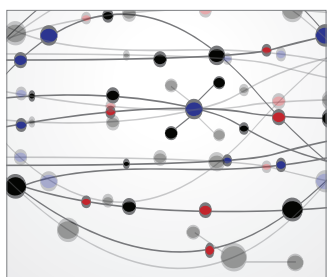

The Scientific World Journal
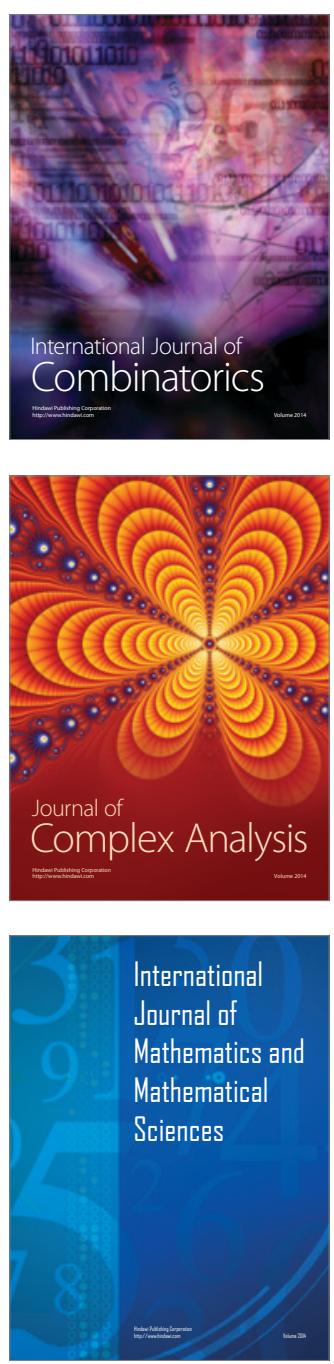
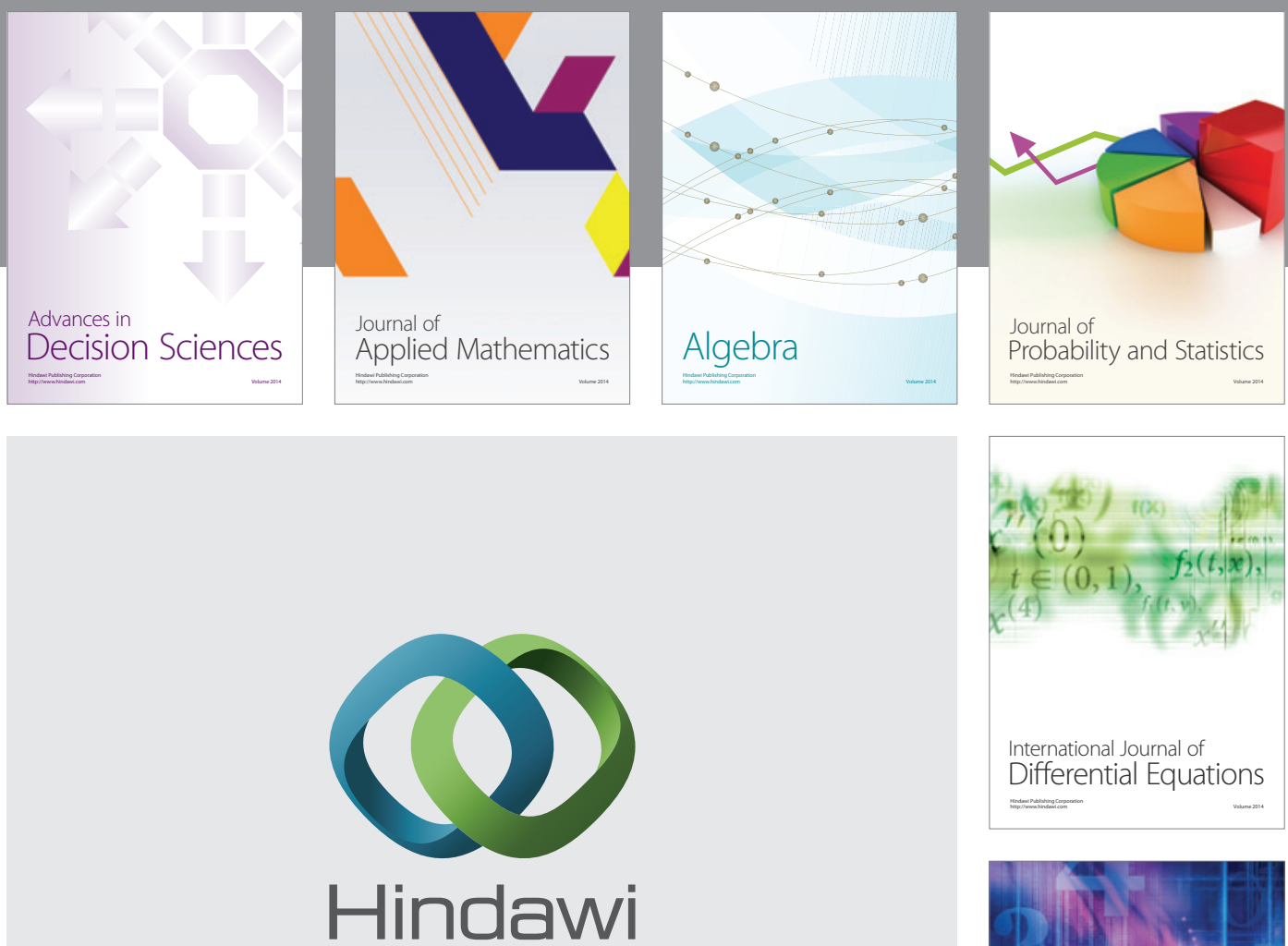

Submit your manuscripts at http://www.hindawi.com
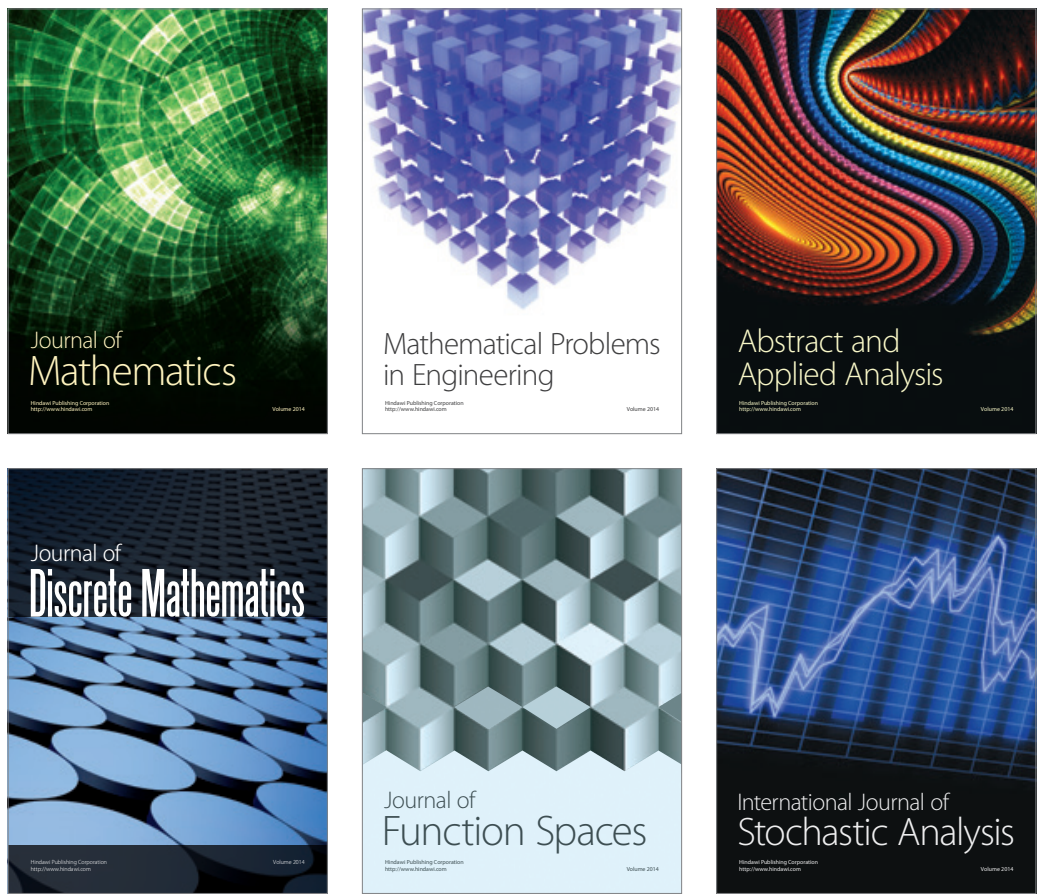

Journal of

Function Spaces

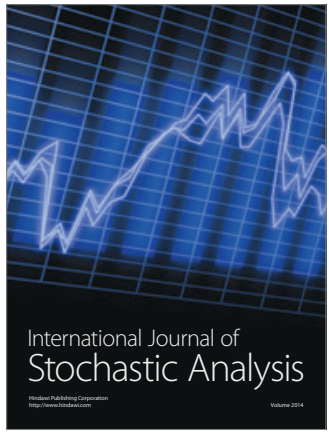

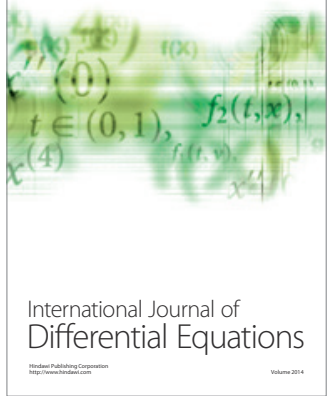
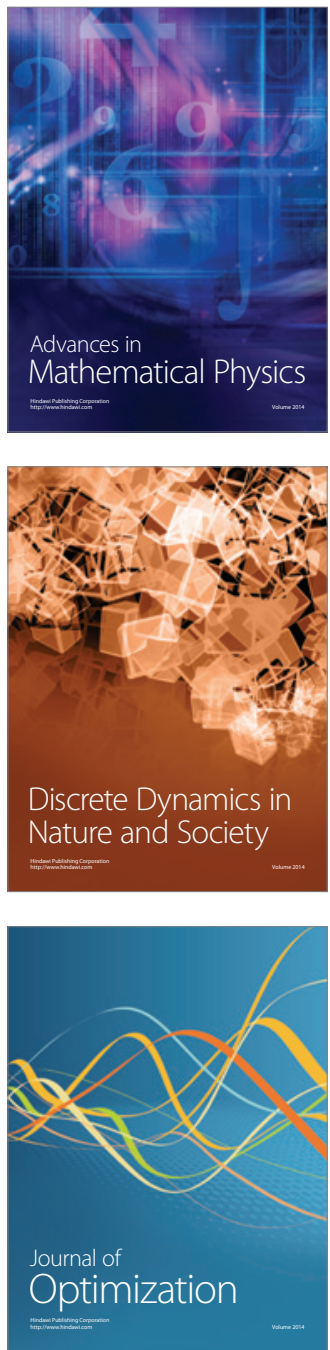